\title{
Diagnostic Concordance and Preoperative Risk Factors for Malignancy in Pancreatic Mucinous Cystic Neoplasms
}

\author{
Ga Hee Kim¹, Kyu Choi', Namyoung Paik ${ }^{1}$, Kyu Taek Lee ${ }^{1}$, Jong Kyun Lee ${ }^{1}$, Kwang Hyuck Lee ${ }^{1}$, In Woong Han², \\ Soo Hoon Kang ${ }^{1}$, Jin Seok $\mathrm{Heo}^{2}$, and Joo Kyung Park ${ }^{1,3}$ \\ Departments of ${ }^{1}$ Medicine and ${ }^{2}$ Surgery, Samsung Medical Center, Sungkyunkwan University School of Medicine, and ${ }^{3}$ Department of \\ Health Sciences and Technology, SAIHST, Sungkyunkwan University, Seoul, Korea
}

\section{Article Info}

Received May 23, 2021

Revised August 24, 2021

Accepted September 3, 2021

Published online December 21, 2021

\section{Corresponding Author \\ Joo Kyung Park \\ ORCID https://orcid.org/0000-0002-9652-5287 \\ E-mail jksophie.park@samsung.com}

\section{Jin Seok Heo}

ORCID https://orcid.org/0000-0001-6767-2790

E-mail jinseok.heo@samsung.com

Ga Hee Kim, Kyu Choi, and Namyoung Paik contributed equally to this work as first authors.
Background/Aims: As pancreatic mucinous cystic neoplasms ( $\mathrm{MCNs}$ ) are considered premalignant lesions, the current guidelines recommend their surgical resection. We aimed to investigate the concordance between preoperative and postoperative diagnoses and evaluate preoperative clinical parameters that could predict the malignant potential of MCNs.

Methods: Patients who underwent surgical resection at Samsung Medical Center for pancreatic cystic lesions and whose pathology was confirmed to be MCN, between July 2000 and December 2017, were retrospectively analyzed.

Results: Among a total of 132 patients $99(75 \%)$ were diagnosed with MCN preoperatively. The most discordant preoperative diagnosis was an indeterminate pancreatic cyst. The proportion of male patients was higher $(24.2 \%$ vs $7.1 \%, p=0.05)$ in the diagnosis-discordance group and the presence of worrisome features in radiologic imaging studies, such as wall thickening/enhancement $(12.1 \%$ vs $37.4 \%, p=0.02)$ or solid component/mural nodule ( $3.0 \%$ vs $27.3 \%, p=0.02)$, was lower in the diagnosis-discordance group. The presence of symptoms ( $57.7 \%$ vs $34.9 \%, p=0.02)$, tumor size greater than $4 \mathrm{~cm}(80.8 \%$ vs $55.7 \%, p=0.04)$, and radiologic presence of a solid component/mural nodule $(42.3 \%$ vs $16.0 \%, p=0.01)$ or duct dilatation $(19.2 \%$ vs $6.6 \%, p=0.01)$ were significantly associated with malignant $\mathrm{MCNs}$.

Conclusions: In our study, the overall diagnostic concordance rate was confirmed to be $75 \%$, and our findings suggest that MCNs have a low malignancy potential when they are less than 4 $\mathrm{cm}$ in size, are asymptomatic and lack worrisome features on preoperative images. (Gut Liver 2022;16:637-644)

Key Words: Mucinous cystadenoma; Mucinous cystadenocarcinoma; Pancreatic neoplasm; Diagnostic accuracy

\section{INTRODUCTION}

With the increased use of advanced cross-sectional imaging modalities and the generalization of health screening examinations, pancreatic cystic neoplasms (PCNs) have been recognized more frequently in clinical practice compared to before. ${ }^{1,2}$ In Korea, the prevalence of incidentally detected PCNs was $2.1 \%$ and the annual incidence of PCNs has increased gradually. ${ }^{1,3}$ The World Health Organization outlines four general categories for the histological classification of PCNs: serous cystic neoplasms, mucinous cystic neoplasms (MCNs), intraductal papillary mucinous neo- plasms (IPMNs), and solid pseudopapillary neoplasms. ${ }^{4,5}$ The biological behavior of these lesions covers a wide spectrum, ranging from benign to borderline and malignant. ${ }^{6}$

Among these lesions, MCNs and IPMNs are known as premalignant lesions. Surgical resection is considered for the management of MCNs and IPMNs with the possibility of malignant transformation. ${ }^{7}$ Recently, some reports indicating MCNs to be less aggressive than previously thought have been emerging. ${ }^{8,9}$ Therefore, making a precise preoperative diagnosis is crucial from the viewpoint of two aspects: risk of over-treatment with unnecessary surgery and risk of under-treatment that would retain a potentially 
malignant tumor. However, there are few studies on the accuracy of preoperative diagnostic assessment. ${ }^{6,10-12}$ Moreover, these past studies focused on overall PCNs.

Although both MCNs and IPMNs are premalignant lesions, MCNs have not been studied in much detail, as opposed to IPMNs. Current international consensus guidelines have also mostly focused on IPMNs. ${ }^{13,14}$ Therefore, in this study, we aimed to investigate the concordance between preoperative and postoperative diagnoses and evaluate preoperative clinical parameters that would predict the malignant potential of MCNs.

\section{MATERIALS AND METHODS}

\section{Patients}

We retrospectively analyzed patients who underwent surgical resection for pancreatic cystic lesions and whose pathology was confirmed to be MCN at Samsung Medical Center between July 2000 and December 2017. All available clinical information including demographic, serum tumor marker, radiologic, surgical, and pathologic data was collected from the medical records.

Preoperative diagnoses were made by clinicians mostly based on imaging studies. All patients had preoperatively undergone at least one cross-sectional imaging study, including computed tomography (CT) or magnetic resonance imaging (MRI). Imaging performed outside the hospital was formally reviewed by the radiologists at our institution. Some of the patients additionally underwent endoscopic ultrasound (EUS), with or without cystic fluid aspiration and/or fine needle aspiration, when cross-sectional imaging was ambiguous. When a patient underwent more than one imaging study and had several diagnostic impressions, the clinician made a high-probability diagnosis considering various factors comprehensively.

Considering the malignant potential and consensus guidelines, ${ }^{13,15,16}$ all patients diagnosed with MCNs preoperatively, underwent surgical resection. In addition, some patients who were not diagnosed with MCNs preoperatively were operated for the following reasons: presence of highly suspected malignant features, increment in the tumor size, diagnostic purpose, presence of symptoms or concomitant complications.

The postoperative pathologic diagnosis was made on the basis of the World Health Organization histological classification. Epithelial dysplasia was graded on the basis of the most severe focus identified. ${ }^{5,17}$ We classified MCNs as benign and malignant in accordance with the histologic grade. Low-grade dysplasia or intermediate-grade dysplasia was considered as a benign lesion and high-grade dysplasia/carcinoma in situ (HGD/CIS) or an invasive mucinous cystadenocarcinoma was considered as a malignant lesion.

The study protocol was reviewed and approved by the Institutional Review Board of Samsung Medical Center (IRB number: 2018-08-006-011). Because of the retrospective design, the informed consent was waived.

\section{Statistical analysis}

Continuous variables were compared using the MannWhitney test. Categorical variables were compared using the chi-square or Fisher exact test. To identify factors associated with diagnostic concordance and malignant MCNs, a univariate binary logistic regression analysis was performed. A multivariable binary logistic regression model was created for variables that were statistically relevant in the univariate analyses. $\mathrm{p}$-values $<0.05$ were considered statistically significant. All statistical analyses were conducted using SPSS Statistics, version 25 (IBM Corp., Armonk, NY, USA).

Table 1. Baseline Characteristics of Patients with Mucinous Cystic Neoplasms

\begin{tabular}{lc}
\hline \multicolumn{1}{c}{ Characteristics } & Value $(n=132)$ \\
\hline Sex & \\
Female & $117(88.6)$ \\
Male & $15(11.4)$ \\
Age, yr & $50(39-60)$ \\
Chief symptom & \\
Asymptomatic & $80(60.6)$ \\
Abdominal pain/discomfort & $40(30.3)$ \\
Palpable mass & $8(6.1)$ \\
Indigestion & $4(3.0)$ \\
Tumor marker & \\
CA19-9, U/mL & $12.8(6.4-32.3)$ \\
Location & \\
Head \& neck & $14(10.6)$ \\
Body & $31(23.5)$ \\
Tail & $87(65.9)$ \\
Size, cm & $4.5(3-8)$ \\
Histologic grade & \\
Low-grade dysplasia & $88(66.7)$ \\
Intermediate-grade dysplasia & $18(13.6)$ \\
High-grade dysplasia/carcinoma in situ & $10(7.6)$ \\
Invasive mucinous cystadenocarcinoma & $16(12.1)$ \\
Surgical procedure & \\
Distal pancreatectomy & $108(81.8)$ \\
Pancreatoduodenectomy & $11(8.3)$ \\
Enucleation & $8(6.1)$ \\
Central pancreatectomy & $3(2.3)$ \\
Subtotal pancreatectomy & $2(1.5)$ \\
\hline Da are & \\
\hline & \\
\hline
\end{tabular}

Data are presented as the number (\%) or median (interquartile range).

CA19-9, carbohydrate antigen 19-9. 


\section{RESULTS}

\section{Patients characteristics}

A total of 132 patients with confirmed histology of MCN after surgical resection were analyzed. Their baseline characteristics are shown in Table 1. The patients were mostly middle-aged (median 50 years) women (88.6\%). More than half of the patients were asymptomatic, but visited our clinic for the evaluation of pancreatic cystic lesions found incidentally. The patients who initially had symptoms complained of abdominal pain/discomfort (30.3\%), palpable masses (6.1\%), and indigestion (3\%).

Most of the tumors were located at the pancreatic body and tail (89.4\%). The median tumor size of the resected specimens was $4.5 \mathrm{~cm}$. In terms of the histologic grade, 88 (66.7\%) patients revealed low-grade dysplasia; 18, intermediate-grade dysplasia (13.6\%); 10, HGD/CIS (7.6\%); and 16 , invasive mucinous cystadenocarcinoma (12.1\%). Most of the patients underwent distal pancreatectomy; other procedures including pancreatoduodenectomy, enucleation, central pancreatectomy, and subtotal pancreatectomy were performed considering the location of the tumor.

For evaluating pancreatic cystic lesions, the patients underwent several imaging studies including CT, MRI,

Table 2. Numbers of Diagnostic Imaging Modalities Performed Preoperatively

\begin{tabular}{cc}
\hline Variable & No. (\%) (n=132) \\
\hline One modality & $63(47.7)$ \\
CT alone & $56(42.4)$ \\
MRI alone & $7(5.3)$ \\
Two modalities & $55(41.7)$ \\
CT+MRI & $25(18.9)$ \\
CT+EUS & $29(22.0)$ \\
MRI+EUS & $1(0.8)$ \\
Three modalities & $14(10.6)$ \\
CT+MRI+EUS
\end{tabular}

CT, computed tomography; MRI, magnetic resonance imaging; EUS, endoscopic ultrasound. and EUS. The numbers of diagnostic imaging modalities performed preoperatively are shown in Table 2 . Among all the patients, 63 (47.7\%) underwent a single imaging study (CT or MRI alone) and 69 (52.3\%) underwent more than two imaging studies before surgical resection.

\section{Diagnostic concordance}

A total of 99 patients were diagnosed with MCNs preoperatively and the overall diagnostic concordance rate was $75 \%$. When the total study period was divided into three parts, the diagnostic concordance rate increased over time as shown in Fig. 1. The detailed information of diagnostic discrepancies is shown in Table 3. The most discordant preoperative diagnosis in patients with MCNs was an indeterminate pancreatic cyst (9.8\%). Other preoperative misdiagnoses were IPMN (3.8\%), solid pseudopapillary neoplasm $(4.5 \%)$, serous cystic neoplasm $(0.8 \%)$, pseudocyst $(2.3 \%)$, pancreatic simple cyst $(0.8 \%)$, pancreatic hemorrhagic cyst $(0.8 \%)$, omental cyst $(0.8 \%)$, left adrenal gland cyst $(0.8 \%)$, and peritoneal origin tumor $(0.8 \%)$. The reasons that the patients with diagnosis dis-

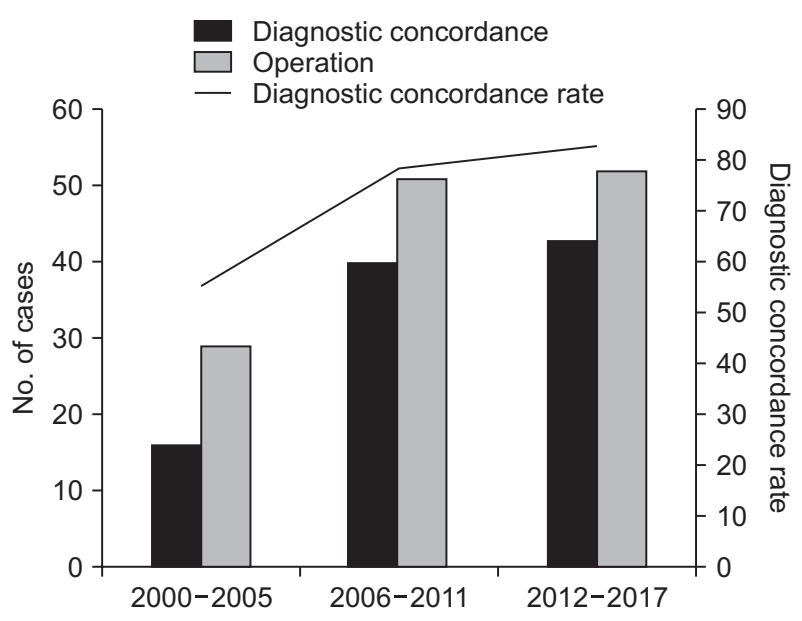

Fig. 1. Diagnostic concordance rate.

Table 3. Comparison between Preoperative and Postoperative Diagnoses

\begin{tabular}{|c|c|c|c|c|c|}
\hline \multirow{3}{*}{ Preoperative diagnosis } & \multirow{3}{*}{$\begin{array}{l}\text { No. }(\%) \\
\text { (n=132) }\end{array}$} & \multicolumn{4}{|c|}{ Postoperative histology } \\
\hline & & \multicolumn{2}{|c|}{ Benign group } & \multicolumn{2}{|c|}{ Malignant group } \\
\hline & & LGD & IGD & $\mathrm{HGD} / \mathrm{CIS}$ & Invasive \\
\hline Mucinous cystic neoplasm & 99 (75.0) & 68 & 10 & 10 & 11 \\
\hline Intraductal papillary mucinous neoplasm & 5 (3.8) & 3 & 1 & - & 1 \\
\hline Solid pseudopapillary neoplasm & $6(4.5)$ & 2 & 2 & - & 2 \\
\hline Serous cystic neoplasm & $1(0.8)$ & 1 & - & - & - \\
\hline Pseudocyst & $3(2.3)$ & 1 & 1 & - & 1 \\
\hline Others & 5 (3.8) & 3 & 2 & - & - \\
\hline Indeterminate & $13(9.8)$ & 10 & 2 & - & 1 \\
\hline
\end{tabular}

LGD, low-grade dysplasia; IGD, intermediate-grade dysplasia; HGD, high-grade dysplasia; CIS, carcinoma in situ. 
cordance underwent operation were as follows: exclusion of malignancy, increment in the tumor size, diagnosis and treatment for symptomatic patients, and presence of concomitant complications such as hemoperitoneum or peritonitis. In terms of the histology, the proportion of patients with invasive mucinous cystadenocarcinoma was $11.1 \%$ in the diagnosis-concordance group. Invasive mucinous cystadenocarcinoma was also diagnosed in the diagnosisdiscordance group and the proportion was $15.2 \%$. Since we defined HGD/CIS as a malignant lesion, 10 patients (10.1\%) considered to have malignancy in the diagnosisconcordance group. However, there was no patient with HGD/CIS in the diagnosis-discordance group.

In the univariate analyses to evaluate factors influencing diagnostic concordance, the following factors were not significant: age, presence of symptoms, serum carbohydrate antigen 19-9 (CA19-9) level, location, size, histologic grade, and number of imaging modalities (Table 4). On the other hand, in the diagnosis-discordance group, the proportion of male patients was higher $(24.2 \%$ vs $7.1 \%$, $\mathrm{p}=0.05$ ) than in the diagnosis-concordance group. In addition, the presence of worrisome features in imaging studies such as wall thickening/enhancement $(12.1 \%$ vs
$37.4 \%, \mathrm{p}=0.04)$ or a solid component/mural nodule $(3.0 \%$ vs $27.3 \%, \mathrm{p}=0.03$ ) was lower in the diagnosis-discordance group. In the multivariable analyses, all of these variables remained as factors independently associated with diagnostic concordance.

\section{Risk factors for $\mathrm{HGD} / \mathrm{CIS}$ or invasive carcinoma in MCNs}

Of all the 132 patients, 26 (19.7\%) were diagnosed with malignant MCNs, including HGD/CIS and invasive mucinous cystadenocarcinoma (Table 5). In the univariate analyses, presence of symptoms $(57.7 \%$ vs $34.9 \%$, $\mathrm{p}=0.006$ ), serum CA19-9 level (median $51.7 \mathrm{U} / \mathrm{mL}$ vs median $11.6 \mathrm{U} / \mathrm{mL}, \mathrm{p}=0.000$ ), tumor size (median $6 \mathrm{~cm}$ vs median $4 \mathrm{~cm}, \mathrm{p}=0.05$ ), tumor size greater than $4 \mathrm{~cm}$ ( $80.8 \%$ vs $55.7 \%, p=0.03)$, and radiologic presence of a solid component/mural nodule $(42.3 \%$ vs $16.0 \%, \mathrm{p}=0.03)$ or duct dilatation $(19.2 \%$ vs $6.6 \%, \mathrm{p}=0.01)$ were significantly associated with malignant MCNs. Among these variables, all except serum CA19-9 level and median tumor size were remained independently associated with malignant MCNs in the multivariable analyses. There was no association of gender, age, location, radiologic presence

Table 4. Factors Influencing Diagnostic Concordance in Mucinous Cystic Neoplasm

\begin{tabular}{|c|c|c|c|c|}
\hline \multirow{2}{*}{ Variable } & \multirow{2}{*}{$\begin{array}{l}\text { Diagnosis-concordance } \\
\text { group ( } \mathrm{n}=99)\end{array}$} & \multirow{2}{*}{$\begin{array}{l}\text { Diagnosis-discordance } \\
\text { group ( } \mathrm{n}=33 \text { ) }\end{array}$} & \multicolumn{2}{|c|}{$\mathrm{p}$-value } \\
\hline & & & Univariate analysis & Multivariable analysis \\
\hline Sex & & & $0.05^{*}$ & $0.05^{*}$ \\
\hline Female & 92 (92.9) & 25 (75.8) & & \\
\hline Male & $7(7.1)$ & $8(24.2)$ & & \\
\hline Age, yr & $51(40-60)$ & $46(33-61)$ & 0.36 & NA \\
\hline Presence of symptoms & & & 0.42 & NA \\
\hline Yes & 36 (36.4) & $16(48.5)$ & & \\
\hline No & $63(63.6)$ & $17(51.5)$ & & \\
\hline Tumor marker & & & 0.31 & NA \\
\hline CA19-9, U/mL & $12.6(6.2-27.1)$ & $14.1(7.1-38.3)$ & & \\
\hline \multicolumn{5}{|l|}{ Location } \\
\hline Head \& neck & $11(11.1)$ & $3(9.1)$ & 0.61 & NA \\
\hline Body & 21 (21.2) & $10(30.3)$ & 0.32 & NA \\
\hline Tail & $67(67.7)$ & $20(60.6)$ & 0.49 & NA \\
\hline Size, cm & $4.5(3.0-8.0)$ & $5.0(2.7-7.6)$ & 0.68 & NA \\
\hline Histologic grade & & & 0.53 & NA \\
\hline LGD/IGD & 78 (78.8) & 28 (84.8) & & \\
\hline HGD/CIS/invasive & $21(21.2)$ & $5(15.2)$ & & \\
\hline \multicolumn{5}{|l|}{ Worrisome features at images } \\
\hline Wall thickening/enhancement & $37(37.4)$ & $4(12.1)$ & $0.04^{*}$ & $0.02 *$ \\
\hline Solid component/mural nodule & 27 (27.3) & $1(3.0)$ & $0.03^{*}$ & $0.02^{*}$ \\
\hline Duct dilatation & $11(11.1)$ & $1(3.0)$ & 0.27 & NA \\
\hline No. of imaging modalities & & & 0.98 & NA \\
\hline One & $43(43.4)$ & $20(60.6)$ & & \\
\hline Two or three & $56(56.6)$ & $13(39.4)$ & & \\
\hline
\end{tabular}

Data are presented as the number (\%) or median (interquartile range).

CA19-9, carbohydrate antigen 19-9; LGD, low-grade dysplasia; IGD, intermediate-grade dysplasia; HGD, high-grade dysplasia; CIS, carcinoma in situ; NA, not available.

*Statistically significant, $p<0.05$. 
Table 5. Risk Factors for High-Grade Dysplasia/Carcinoma In Situ or Invasive Carcinoma in Mucinous Cystic Neoplasms

\begin{tabular}{|c|c|c|c|c|c|}
\hline \multirow{2}{*}{ Variable } & \multirow{2}{*}{$\begin{array}{l}\text { Benign group } \\
\quad(n=106)\end{array}$} & \multirow{2}{*}{$\begin{array}{l}\text { Malignant group } \\
\qquad(\mathrm{n}=26)\end{array}$} & \multirow{2}{*}{$\frac{\text { Univariate analysis }}{\mathrm{p} \text {-value }}$} & \multicolumn{2}{|c|}{ Multivariable analysis } \\
\hline & & & & OR $(95 \% \mathrm{Cl})$ & p-value \\
\hline Sex & & & 0.21 & NA & NA \\
\hline Female & $95(89.6)$ & $22(84.6)$ & & & \\
\hline Male & $11(10.4)$ & $4(15.4)$ & & & \\
\hline Age, yr & $49(38-58)$ & $53(40-61)$ & 0.15 & NA & NA \\
\hline Presence of symptoms & & & $0.01 *$ & $3.93(1.29-12.03)$ & $0.02 *$ \\
\hline Yes & 37 (34.9) & 15 (57.7) & & & \\
\hline No & $69(65.1)$ & $11(42.3)$ & & & \\
\hline Tumor marker & & & $0.00 *$ & $1.00(1.00-1.00)$ & 0.40 \\
\hline CA19-9, U/mL & $11.6(6.0-21.6)$ & $51.7(11.3-2,026.3)$ & & & \\
\hline \multicolumn{6}{|l|}{ Location } \\
\hline Head \& neck & $10(9.4)$ & $4(15.4)$ & 0.98 & NA & NA \\
\hline Body & $25(23.6)$ & $6(23.1)$ & 0.98 & NA & NA \\
\hline Tail & $71(67.0)$ & $16(61.5)$ & 0.92 & NA & NA \\
\hline Median size, $\mathrm{cm}$ & $4(3-8)$ & $6(4-8)$ & $0.05^{*}$ & $0.90(0.75-1.07)$ & 0.23 \\
\hline Size & & & $0.03 *$ & $3.97(1.04-15.21)$ & $0.04^{*}$ \\
\hline$<4 \mathrm{~cm}$ & $47(44.3)$ & $5(19.2)$ & & & \\
\hline$\geq 4 \mathrm{~cm}$ & $59(55.7)$ & $21(80.8)$ & & & \\
\hline \multicolumn{6}{|l|}{ Worrisome features at images } \\
\hline Wall thickening/enhancement & $30(28.3)$ & $11(42.3)$ & 0.52 & NA & NA \\
\hline Solid component/mural nodule & $17(16.0)$ & $11(42.3)$ & $0.03^{*}$ & $4.59(1.42-14.85)$ & $0.01 *$ \\
\hline Duct dilatation & $7(6.6)$ & 5 (19.2) & $0.01 *$ & $8.54(1.75-14.58)$ & $0.01 *$ \\
\hline No. of imaging modalities & & & 0.31 & NA & NA \\
\hline One & $53(50.0)$ & 10 (38.5) & & & \\
\hline Two or three & $53(50.0)$ & $16(61.5)$ & & & \\
\hline
\end{tabular}

Data are presented as number (\%) or median (interquartile range).

OR, odds ratio; $\mathrm{Cl}$, confidence interval; CA19-9, carbohydrate antigen 19-9; NA, not available.

*Statistically significant, $p<0.05$.

of wall thickening/enhancement, or number of imaging modalities with malignant MCNs.

\section{DISCUSSION}

The number of pancreatic cystic lesions has recently increased. The increased use of cross-sectional imaging modalities and the improved resolution of these modalities have enabled the detection of many incidental lesions. Previous studies in Korea reported the prevalence of PCNs to range from $0.47 \%$ to $2.1 \%{ }^{1,18}$ Among pathology-confirmed PCNs, either surgically or non-surgically (proven by either biopsy or cytological examination), the mucinous type neoplasms such as IPMNs (41.0\%) and MCNs (25.2\%) were more common than the non-mucinous type including solid pseudopapillary neoplasms (18.3\%) and serous cystic neoplasms (15.2\%). Since both IPMNs and MCNs have the possibility of malignant transformation, the international consensus guidelines have proposed surgical resection for mucinous type neoplasms. Notably, the management of IPMNs continues to evolve and the indications for the resection of branch duct IPMNs have recently been revised in detail. ${ }^{14}$ For the management of MCNs however, these same guidelines have constantly recommended surgical resection for all patients with MCNs without any risk stratifications. Only lately has the European Study Group proposed that presumed MCNs without concerning features ( $\geq 40 \mathrm{~mm}$, presence of symptoms, or presence of mural nodules) could be followed up carefully by MRI, EUS, or a combination of both. ${ }^{9}$ Despite the aggressive treatment strategy, the diagnostic accuracy and risk factors for malignancy of MCNs have not been studied as well as those for IPMNs. Indeed, the accurate preoperative diagnosis of each type of PCN is very important because this would determine the treatment strategy. However, accurately diagnosing MCNs preoperatively remains challenging. Only few reports currently exist on the preoperative diagnostic assessment of MCNs. Even if data on this specific topic are limited, the accuracy of cross-sectional imaging modalities for MCNs ranges from $60 \%$ to $79.7 \%{ }^{6,10,11}$ To overcome the imperfection of preoperative diagnosis, various additional techniques including EUS-guided cystic fluid analysis, fine needle aspiration, and even molecular work have been performed. ${ }^{19}$ In addition, little is known about the factors associated with malignant MCNs. To the best of our knowledge, a few studies have evaluated the preoperative risk factors for MCN malignancy, to date. ${ }^{7,9}$ 
Thus, we first investigated the concordance between preoperative and postoperative diagnoses for identifying the diagnostic accuracy of MCNs, in this study. Since the final diagnosis depends on histology after surgical resection, we had to analyze only patients who were confirmed to have MCNs in the postoperative pathology and use the term "concordance" instead of "accuracy." We found that the overall diagnostic concordance rate was $75 \%$, which was in the range previously reported by other studies. Considering that most discordant cases were indeterminate cysts $(9.8 \%)$, the concordance of diagnosis was quite high in this study. In our data, male sex or the absence of worrisome radiologic features such as wall thickening/enhancement or a solid component/mural nodule was associated with diagnostic discordance. The reason for this could be that these factors did not match the traditional features of MCNs. Notably, in this study, the proportion of male patients was relatively higher than in past reports regarding the occurrence of MCNs in men as controversial. ${ }^{20-22} \mathrm{Be}-$ cause we ascertained the presence of ovarian-type stroma in all male patients with MCNs in our study, we believe that MCNs can occur in male patients. Therefore, we think that clinicians should not rule out MCNs in male patients although their probability is low.

Secondly, we evaluated the risk factors that would predict malignant potential preoperatively in patients with MCNs. Malignant MCNs including HGD/CIS and invasive mucinous cystadenocarcinoma were present in $19.7 \%$. Previous studies which included HGD/CIS as malignancy, as in our study, reported this ratio to range from $14.9 \%$ to $17.3 \%$. ${ }^{7.22}$ We found that the presence of symptoms or worrisome radiologic features such as duct dilatation or a solid component/mural nodule was associated with malignancy. Postlewait et al. ${ }^{7}$ recently reported male sex, a pancreatic head and neck location, larger MCNs, a solid component or mural nodule, and duct dilatation as preoperative risk factors. Unlike the studies that included postoperative pathological parameters, their study was noteworthy in they dealt with only preoperative clinical parameters. Compared to their study, the proportion of malignant MCNs was higher in our study. In terms of risk factors, the radiologic presence of duct dilatation or a solid component/mural nodule was also associated with malignancy, as in their study. Instead of sex, location, or size, however, the presence of symptoms was significantly associated with malignant MCNs.

Recently, the European Study Group published the European evidence-based guidelines. ${ }^{9}$ The differences in the management of MCNs from the international consensus guidelines were that instead of recommending surgical resection for all patients diagnosed with MCNs, those with a size of MCN less than $4 \mathrm{~cm}$ and without any symptoms or presence of worrisome features such as a mural nodule could be observed for progress. A meta-analysis reported by Nilsson et al. ${ }^{8}$ provided background evidence, and according to their report, MCNs are probably more indolent lesions than were previously thought and among those less than $4 \mathrm{~cm}$ in size, only $0.03 \%$ were associated with invasive cystadenocarcinoma. Likewise, the presence of symptoms or worrisome radiologic features, but not size, was associated with malignant MCNs, in our study. To validate the suitability of this size criterion, we additionally analyzed the association between malignancy and size based on the criterion of $4 \mathrm{~cm}$, and found that a size of $4 \mathrm{~cm}$ or more was significantly associated with malignancy. Five patients (19.2\%) were diagnosed with malignant MCNs with sizes less than $4 \mathrm{~cm}$. Among them, two were diagnosed with an invasive cystadenocarcinoma and three with HGD/CIS. All patients with invasive cancer had both, a size more than 3 $\mathrm{cm}$ and the radiologic presence of a solid portion or mural nodule. These findings in our study support the recent $\mathrm{Eu}$ ropean guidelines for the more conservative management of MCNs.

This single-center retrospective observational study has some limitations. The study subjects were limited to patients who underwent surgery. Although this was inevitable to find a definite MCN, there might have been bias in selection because it excluded patients with MCNs who did not undergo surgical resection. In addition, not all subjects received the same imaging tests and this might potentially affect the diagnostic accuracy. Because the target study period was long, the images were read by a large number of radiologists and endoscopists but we did not review the imaging studies again for objectification. Finally, patients evaluated only by CT imaging before surgery accounted for the largest proportion (42.4\%). Magnetic resonance cholangiopancreatography is more sensitive than CT for identifying communication between the PCN and the pancreatic duct system and the presence of mural nodule or internal septations, and EUS is also used to identify additional features when considering surgical resection of PCN. ${ }^{9} \mathrm{CT}$ alone might be insufficient for preoperative evaluation, and as mentioned above, it might affect diagnostic accuracy.

In conclusion, the overall diagnostic concordance rate of $75 \%$ in patients with MCNs was quite high in our study. Male sex or the absence of worrisome radiologic features such as wall thickening/enhancement or a solid component/mural nodule was associated with diagnostic discordance. Moreover, our study supports the emerging trends in the literature that MCNs have low malignant potential when they are smaller than $4 \mathrm{~cm}$, asymptomatic and when 
there is a lack of worrisome features on preoperative imaging.

\section{CONFLICTS OF INTEREST}

No potential conflict of interest relevant to this article was reported.

\section{AUTHOR CONTRIBUTIONS}

Study concept and design: J.K.P. Acquisition, analysis, or interpretation of data: G.H.K., K.C., N.P. Writing and drafting of the manuscript: G.H.K., K.C., N.P. Critical revision of the manuscript for important intellectual content: K.T.L., J.K.L., K.H.L., J.S.H., I.W.H., S.H.K. All authors approved the final submission.

\section{ORCID}

Ga Hee Kim https://orcid.org/0000-0002-7214-9918

Kyu Choi https://orcid.org/0000-0001-5147-1409

Namyoung Paik https://orcid.org/0000-0002-3718-7433

Kyu Taek Lee https://orcid.org/0000-0003-2233-3511

Jong Kyun Lee https://orcid.org/0000-0002-9384-3079

Kwang Hyuck Lee https://orcid.org/0000-0002-2898-6233

In Woong Han https://orcid.org/0000-0001-7093-2469

Soo Hoon Kang https://orcid.org/0000-0001-6250-0594

Jin Seok Heo https://orcid.org/0000-0001-6767-2790

Joo Kyung Park https://orcid.org/0000-0002-9652-5287

\section{REFERENCES}

1. Chang YR, Park JK, Jang JY, Kwon W, Yoon JH, Kim SW. Incidental pancreatic cystic neoplasms in an asymptomatic healthy population of 21,745 individuals: large-scale, singlecenter cohort study. Medicine (Baltimore) 2016;95:e5535.

2. de Jong K, Nio CY, Hermans JJ, et al. High prevalence of pancreatic cysts detected by screening magnetic resonance imaging examinations. Clin Gastroenterol Hepatol 2010;8:806-811.

3. Yoon WJ, Lee JK, Lee KH, Ryu JK, Kim YT, Yoon YB. Cystic neoplasms of the exocrine pancreas: an update of a nationwide survey in Korea. Pancreas 2008;37:254-258.

4. Chiang AL, Lee LS. Clinical approach to incidental pancreatic cysts. World J Gastroenterol 2016;22:1236-1245.

5. Zamboni G, Longnecker DS, Klöppel G, Adler G, Hruban RH. Mucinous cystic neoplasms of the pancreas. In: Hamil- ton SR, Aaltonen LA, eds. World Health Organization classification of tumours: pathology and genetics of tumours of the digestive system. Lyon: IARC Press, 2000:234-236.

6. Del Chiaro M, Segersvärd R, Pozzi Mucelli R, et al. Comparison of preoperative conference-based diagnosis with histology of cystic tumors of the pancreas. Ann Surg Oncol 2014;21:1539-1544.

7. Postlewait LM, Ethun CG, McInnis MR, et al. Association of preoperative risk factors with malignancy in pancreatic mucinous cystic neoplasms: a multicenter study. JAMA Surg 2017;152:19-25.

8. Nilsson LN, Keane MG, Shamali A, et al. Nature and management of pancreatic mucinous cystic neoplasm (MCN): a systematic review of the literature. Pancreatology 2016;16:1028-1036.

9. European Study Group on Cystic Tumours of the Pancreas. European evidence-based guidelines on pancreatic cystic neoplasms. Gut 2018;67:789-804.

10. Correa-Gallego C, Ferrone CR, Thayer SP, Wargo JA, Warshaw AL, Fernández-Del Castillo C. Incidental pancreatic cysts: do we really know what we are watching? Pancreatology 2010;10:144-150.

11. Salvia R, Malleo G, Marchegiani G, et al. Pancreatic resections for cystic neoplasms: from the surgeon's presumption to the pathologist's reality. Surgery 2012;152(3 Suppl 1):S135-S142.

12. Cho CS, Russ AJ, Loeffler AG, et al. Preoperative classification of pancreatic cystic neoplasms: the clinical significance of diagnostic inaccuracy. Ann Surg Oncol 2013;20:31123119.

13. Tanaka M, Fernández-del Castillo C, Adsay V, et al. International consensus guidelines 2012 for the management of IPMN and MCN of the pancreas. Pancreatology 2012;12:183-197.

14. Tanaka M, Fernández-Del Castillo C, Kamisawa T, et al. Revisions of international consensus Fukuoka guidelines for the management of IPMN of the pancreas. Pancreatology 2017;17:738-753.

15. Tanaka M, Chari S, Adsay V, et al. International consensus guidelines for management of intraductal papillary mucinous neoplasms and mucinous cystic neoplasms of the pancreas. Pancreatology 2006;6:17-32.

16. Vege SS, Ziring B, Jain R, Moayyedi P; Clinical Guidelines Committee; American Gastroenterology Association. American gastroenterological association institute guideline on the diagnosis and management of asymptomatic neoplastic pancreatic cysts. Gastroenterology 2015;148:819-822.

17. Jang KT, Park SM, Basturk O, et al. Clinicopathologic characteristics of 29 invasive carcinomas arising in 178 pancreatic mucinous cystic neoplasms with ovarian-type stroma: implications for management and prognosis. Am J Surg 
Pathol 2015;39:179-187.

18. Lee SH, Shin CM, Park JK, et al. Outcomes of cystic lesions in the pancreas after extended follow-up. Dig Dis Sci 2007;52:2653-2659.

19. Springer S, Wang Y, Dal Molin M, et al. A combination of molecular markers and clinical features improve the classification of pancreatic cysts. Gastroenterology 2015;149:15011510.

20. Reddy RP, Smyrk TC, Zapiach M, et al. Pancreatic mucinous cystic neoplasm defined by ovarian stroma: demographics, clinical features, and prevalence of cancer. Clin Gastroenterol Hepatol 2004;2:1026-1031.

21. Suzuki Y, Atomi Y, Sugiyama M, et al. Cystic neoplasm of the pancreas: a Japanese multiinstitutional study of intraductal papillary mucinous tumor and mucinous cystic tumor. Pancreas 2004;28:241-246.

22. Yamao K, Yanagisawa A, Takahashi K, et al. Clinicopathological features and prognosis of mucinous cystic neoplasm with ovarian-type stroma: a multi-institutional study of the Japan pancreas society. Pancreas 2011;40:67-71. 